\title{
Delayed Age Replacement Policy with Uncertain Lifetime
}

\author{
Xueyan Li and Chunxiao Zhang \\ College of Science, Civil Aviation University of China, Tianjin 300300, China \\ Correspondence should be addressed to Xueyan Li; zh83665727@126.com
}

Received 4 July 2014; Accepted 11 October 2014

Academic Editor: Wenbin Wang

Copyright (C) $2015 \mathrm{X}$. Li and C. Zhang. This is an open access article distributed under the Creative Commons Attribution License, which permits unrestricted use, distribution, and reproduction in any medium, provided the original work is properly cited.

\begin{abstract}
This paper considers the delayed age replacement policy, in which the lifetimes of all units are assumed to be uncertain variables, and the lifetime of the first unit has an uncertainty distribution which is different from the others. A delayed age replacement model which is concerned with finding the optimal replacement time to minimize the expected cost is developed. In the policy, the optimal replacement time is irrelevant to the uncertain distribution of lifetime of the first unit over the infinite time span.
\end{abstract}

\section{Introduction}

The replacement policy for the unit based on its age is called age replacement policy, which means that a unit is always replaced at failure or at an age $T$, whichever occurs first. Age replacement policy is easy to operate especially for multicomponent systems, so it is one of the widely used maintenance policies. Age replacement policies have been studied theoretically by many authors. In 1965, Barlow and Proschan [1] studied the basic replacement policies. Furthermore, age replacement policy with continuous discounting was proposed by Fox in 1966 [2]. Scheaffer [3] considered optimum age replacement policies with an increasing cost factor. Later in 1979, Cleroux et al. [4] studied age replacement policy with random charges. Cleroux and Hanscom [5] studied a general age replacement model with minimal repair. Boland and Proschan [6] studied the case when the repair cost increases with age. Jhang and Sheu [7] proposed an opportunitybased age replacement policy with minimal repair. For more development of replacement policies, readers can refer to Nakagawa [8].

In the above literatures, the lifetime of a unit is regarded as a random variable, and probability theory is employed to deal with the optimization of the replacement policy. The probability theory is applicable only when we have the large enough sample size. However when no samples are available in some situation, we have to invite some domain experts to evaluate the belief degree that each event will occur. Since human tends to overweight unlikely events [9], the belief degree may have a much larger range than the real frequency. Therefore, it is unreasonable to employ stochastic method for the particularity of the problem. In order to rationally deal with belief degrees, uncertainty theory was founded by Liu in 2007 [10], and was refined by Liu in 2010 [11] based on normality, duality, subadditivity, and product axioms. Nowadays, uncertainty theory has become a branch of axiomatic mathematics for modeling human uncertainty, and some applications can be found in various fields.

Yao and Ralescu [12] firstly proposed the uncertain age replacement policy, where the lifetimes of all units are assumed as iid uncertain variables. However, in practice of maintenance engineering, the lifetime of the first unit may be quite different from the remains. If the age $T$ is unchanging in the age replacement policy, only the lifetime of the first unit has an uncertainty distribution which is different from the others. The first replacement point may be observed at a delayed time and call it delayed age replacement policy as the delayed renewal process. In this paper, we will consider the delayed age replacement policy with uncertain lifetimes of all units, and the lifetime of first unit has a different uncertainty distribution from the others. And then, a delayed age replacement model to find the optimal predetermined replacement time $T$ will be developed.

This paper is organized as follows: Section 2 recalls some basic concepts and properties about uncertainty theory which will be used throughout the paper. In Section 3, delayed age replacement policy in uncertain environment is introduced and the expected cost over infinite time is proposed; thus 
the optimal age replacement time will be derived. Numerical example is given in Section 4 , followed by Section 5 where we conclude the paper.

\section{Preliminaries}

Let $\Gamma$ be a nonempty set. $\mathscr{L}$ is a $\sigma$-algebra on $\Gamma$. Each element $\Lambda$ in the $\sigma$-algebra $\mathscr{L}$ is called an event. Uncertain measure is a function from $\mathscr{L}$ to $[0,1]$. In order to present an axiomatic definition of uncertain measure, it is necessary to assign to each event $\Lambda$ a number $\mathscr{M}\{\Lambda\}$ which indicates the belief degree that the event $\Lambda$ will occur. In order to ensure that the number $\mathscr{M}\{\Lambda\}$ has certain mathematical properties, Liu [10] proposed the following three axioms.

Axiom 1: (normality axiom) $\mathscr{M}\{\Gamma\}=1$ for the universal set $\Gamma$.

Axiom 2: (duality axiom) $\mathscr{M}\{\Lambda\}+\mathscr{M}\left\{\Lambda^{c}\right\}=1$ for any event $\Lambda$.

Axiom 3: (subadditivity axiom) For every countable sequence of events $\Lambda_{1}, \Lambda_{2}, \ldots$, we have

$$
\mathscr{M}\left\{\bigcup_{i=1}^{\infty} \Lambda_{i}\right\} \leq \sum_{i=1}^{\infty} \mathscr{M}\left\{\Lambda_{i}\right\}
$$

Definition 1 (Liu [10]). The set function $\mathscr{M}$ is called an uncertain measure if it satisfies the normality, duality, subadditivity, and product axioms.

Definition 2 (Liu [10]). An uncertain variable is a measurable function $\xi$ from the uncertainty space $(\Gamma, \mathscr{L}, \mathscr{M})$ to the set of real numbers; that is, for any Borel set $B$ of real numbers, the set

$$
\{\xi \in B\}=\{\gamma \in \Gamma \mid \xi(\gamma) \in B\}
$$

is an event.

In order to describe an uncertain variable, a concept of uncertainty distribution is introduced as follows.

Definition 3 (Liu [10]). The uncertainty distribution of an uncertain variable $\xi$ is defined by

$$
\Phi(x)=\mathscr{M}\{\xi \leq x\},
$$

for any real number $x$.

Expected value is the average of an uncertain variable in the sense of uncertain measure and represented the size of uncertain variable.

Definition 4 (Liu [10]). Let $\xi$ be an uncertain variable. Then the expected value of $\xi$ is defined by

$$
\begin{aligned}
E[\xi] & =\int_{0}^{+\infty} \mathscr{M}\{\xi \geq r\} d r-\int_{-\infty}^{0} \mathscr{M}\{\xi \leq r\} d r \\
& =\int_{0}^{+\infty}(1-\Phi(x)) d x-\int_{-\infty}^{0} \Phi(x) d x
\end{aligned}
$$

An uncertain process [13] is essentially a sequence of uncertain variables indexed by time. Renewal process is one of the most important uncertain processes in which events occur continuously and independently of one another in uncertain times.

Definition 5 (Liu [13]). Let $\xi_{1}, \xi_{2}, \ldots$ be iid positive uncertain variables. Define $S_{0}=0$ and $S_{n}=\xi_{1}+\xi_{2}+\cdots+\xi_{n}$ for $n \geq 1$. Then the uncertain process

$$
N_{t}=\max _{n \geq 0}\left\{n \mid S_{n} \leq t\right\}
$$

is called an uncertain renewal process.

Age replacement means that an element is always replaced at failure or at an age $T$. If $\xi_{1}, \xi_{2}, \ldots$ denote the lifetimes of the elements which are iid uncertain variable with a common uncertainty distribution, then the actual lifetimes of the elements are iid uncertain variables

$$
\xi_{1} \wedge T, \xi_{2} \wedge T, \ldots
$$

which may generate an uncertain renewal process:

$$
N_{t}=\max _{n \geq 0}\left\{n \mid \sum_{i=1}^{n}\left(\xi_{i} \wedge T\right) \leq t\right\} .
$$

Yao and Ralescu [12] investigated the uncertain age replacement policy and obtained the long-run average replacement cost as follows:

$$
\lim _{t \rightarrow \infty} E\left[\sum_{i=1}^{N_{t}} \frac{f\left(\xi_{i} \wedge T\right)}{t}\right]=\frac{a}{T}+\frac{b-a}{T} \Phi(T)+\int_{0}^{T} \frac{\Phi(x)}{x^{2}} d x,
$$

where $f(x)$ is the replacement cost function.

\section{Delayed Age Replacement Policy}

Consider an age replacement policy in which a unit is replaced at constant time $T$ after its installation or at failure, whichever occurs first. We assume that failures are instantly detected and replaced with a new one, where its replacement time is negligible. Assume that the lifetimes of the units are uncertain variables $\xi_{1}, \xi_{2}, \ldots$, and $\xi_{1}$ has an uncertainty distribution which is different from the others. A net unit is installed at time $t=0$. Then, the actual lifetimes of the units are uncertain variables $\xi_{1} \wedge T, \xi_{2} \wedge T, \ldots$, which generate an uncertain delayed renewal process:

$$
N_{t}=\max \left\{n \mid \sum_{i=1}^{n}\left(\xi_{i} \wedge T\right) \leq t\right\},
$$

where $\xi_{1} \wedge T$ has an uncertainty distribution which is different from the others. We consider the problem of minimizing the expected cost per unit of time for an infinite time span. For simplicity, we introduce the following cost function:

$$
f(x)= \begin{cases}b, & \text { if } x<T \\ a, & \text { if } x=T\end{cases}
$$


where $a>0$ is the cost of replacing the unit at age $T$ and $b$ is the cost of replacing the unit at failure, which is larger than $a$. Then $f\left(\xi_{i} \wedge T\right)$ denotes the cost to replace the $i$ th unit, and the expected total replacement cost before time $t$ is

$$
E\left[\sum_{i=1}^{N_{t}} f\left(\xi_{i} \wedge T\right)\right]
$$

The average cost over the time $t$ is expressed as

$$
\lim _{t \rightarrow \infty} E\left[\sum_{i=1}^{N_{t}} \frac{f\left(\xi_{i} \wedge T\right)}{t}\right]
$$

Delayed age replacement policy aims at finding an optimal time $T^{*}$ to minimize the average replacement cost; that is,

$$
\lim _{t \rightarrow \infty} E\left[\sum_{i=1}^{N_{t}} \frac{f\left(\xi_{i} \wedge T^{*}\right)}{t}\right]=\min _{T} \lim _{t \rightarrow \infty} E\left[\sum_{i=1}^{N_{t}} \frac{f\left(\xi_{i} \wedge T\right)}{t}\right] .
$$

Lemma 6 (Yao and Ralescu [12]). Let $\xi$ be a positive uncertain variable with an uncertainty distribution $\Phi$. Given that

$$
f(x)= \begin{cases}b, & \text { if } x<T \\ a, & \text { if } x=T\end{cases}
$$

with $0<a<b$, the uncertain variable

$$
\frac{f(\xi \wedge T)}{\xi \wedge T}
$$

has an uncertainty distribution

$$
\Psi(x)= \begin{cases}0 & \text { if } x<\frac{a}{T} \\ 1-\Phi(T) & \text { if } \frac{a}{T} \leq x<\frac{b}{T}, \\ 1-\Phi\left(\frac{b}{x}\right) & \text { if } x \geq \frac{b}{T} .\end{cases}
$$

Lemma 7 (Yao and Ralescu [12]). Let $N_{t}$ be an uncertain renewal process with iid uncertain interarrival times $\xi_{1}, \xi_{2}, \ldots$, and let $f$ be a positive function. Then

$$
\frac{\sum_{i=1}^{N_{t}} f\left(\xi_{i}\right)}{\sum_{i=1}^{N_{t}} g\left(\xi_{i}\right)}, \quad \frac{f\left(\xi_{1}\right)}{g\left(\xi_{1}\right)}
$$

have a common uncertainty distribution.

Theorem 8. Let $\xi_{1}, \xi_{2}, \ldots$ be a sequence of positive uncertain variables. If $\xi_{1}$ has an uncertainty distribution $\Phi_{1}$ and $\xi_{2}, \xi_{3}, \ldots$ has a common uncertainty distribution $\Phi, N_{t}$ is an uncertain renewal process with uncertain interarrivals $\xi_{1} \wedge T, \xi_{2} \wedge T, \ldots$. Given that

$$
f(x)= \begin{cases}b, & \text { if } x<T \\ a, & \text { if } x=T\end{cases}
$$

with $0<a<b$, the uncertainty distribution $\Psi_{t}(x)$ of the uncertain variable

$$
\sum_{i=1}^{N_{t}} \frac{f\left(\xi_{i} \wedge T\right)}{t}
$$

satisfies

$$
\lim _{t \rightarrow \infty} \Psi_{t}(x) \geq \begin{cases}0 & \text { if } x \leq \frac{a}{T} \\ 1-\Phi(T) & \text { if } \frac{a}{T}<x \leq \frac{b}{T} \\ 1-\Phi\left(\frac{b}{x}\right) & \text { if } x>\frac{b}{T} .\end{cases}
$$

Proof. Since

$$
f\left(\xi_{1} \wedge T\right) \geq a, \quad \sum_{i=2}^{N_{t}}\left(\xi_{i} \wedge T\right) \leq t,
$$

we have

$$
\begin{aligned}
\Psi_{t}(x) & =\mathscr{M}\left\{\sum_{i=1}^{N_{t}} \frac{f\left(\xi_{i} \wedge T\right)}{t} \leq x\right\} \\
& \geq \mathscr{M}\left\{\frac{\left(a+\sum_{i=2}^{N_{t}} f\left(\xi_{i} \wedge T\right)\right)}{t} \leq x\right\} \\
& =\mathscr{M}\left\{\left(\frac{\sum_{i=2}^{N_{t}} f\left(\xi_{i} \wedge T\right)}{\sum_{i=2}^{N_{t}} \xi_{i} \wedge T}\right)\left(\sum_{i=2}^{N_{t}} \frac{\xi_{i} \wedge T}{t}\right) \leq x-\frac{a}{t}\right\} \\
& \geq \mathscr{M}\left\{\frac{\sum_{i=2}^{N_{t}} f\left(\xi_{i} \wedge T\right)}{\sum_{i=2}^{N_{t}}\left(\xi_{i} \wedge T\right)} \leq x-\frac{a}{t}\right\} \\
& =\mathscr{M}\left\{\frac{f\left(\xi_{2} \wedge T\right)}{\xi_{2} \wedge T} \leq x-\frac{a}{t}\right\} \\
& =\Psi\left(x-\frac{a}{t}\right) \quad \text { if } x<\frac{a}{t}+\frac{a}{T}+\frac{a}{T} \leq x<\frac{a}{t}+\frac{b}{T} \\
& = \begin{cases}0 \quad \text { if } x \geq \frac{a}{t}+\frac{b}{T} . \\
1-\Phi\left(\frac{b}{x-a / t}\right.\end{cases}
\end{aligned}
$$

The last second inequality holds because of Lemma 6 .

Thus

$$
\begin{aligned}
\lim _{t \rightarrow \infty} \Psi_{t}(x) & \geq \lim _{t \rightarrow \infty} \Psi\left(x-\frac{a}{t}\right) \\
& = \begin{cases}0 & \text { if } x \leq \frac{a}{T} \\
1-\Phi(T) & \text { if } \frac{a}{T}<x \leq \frac{b}{T} \\
1-\Phi\left(\frac{b}{x}\right) & \text { if } x>\frac{b}{T} .\end{cases}
\end{aligned}
$$

The theorem is proved. 
Theorem 9. Let $\xi_{1}, \xi_{2}, \ldots$ be a sequence of positive uncertain variables. If $\xi_{1}$ has an uncertainty distribution $\Phi_{1}$ and $\xi_{2}, \xi_{3}, \ldots$ has a common uncertainty distribution $\Phi, N_{t}$ is an uncertain renewal process with uncertain interarrivals $\xi_{1} \wedge T, \xi_{2} \wedge T, \ldots$.. Given that

$$
f(x)= \begin{cases}b, & \text { if } x<T \\ a, & \text { if } x=T,\end{cases}
$$

with $0<a<b$ the uncertainty distribution $\Psi_{t}(x)$ of the uncertain variable

$$
\sum_{i=1}^{N_{t}} \frac{f\left(\xi_{i} \wedge T\right)}{t}
$$

satisfies

$$
\lim _{t \rightarrow \infty} \Psi_{t}(x) \leq \begin{cases}0 & \text { if } x<\frac{a}{T} \\ 1-\Phi(T) & \text { if } \frac{a}{T} \leq x<\frac{b}{T} \\ 1-\Phi\left(\frac{b}{x}\right) & \text { if } x \geq \frac{b}{T} .\end{cases}
$$

Proof.

Case 1. Assume that $x<a / T$. Let $t>T a /(a-T x)$; then we have

$$
\begin{aligned}
\sum_{i=1}^{N_{t}} \frac{f\left(\xi_{i} \wedge T\right)}{t} & \geq \frac{a N_{t}}{t} \geq \frac{a(t / T-1)}{t} \\
& =\frac{a}{T}\left(1-\frac{T}{t}\right) \\
& >x .
\end{aligned}
$$

It is concluded that

$$
\lim _{t \rightarrow \infty} \Psi_{t}(x)=\mathscr{M}\left\{\sum_{i=1}^{N_{t}} \frac{f\left(\xi_{i} \wedge T\right)}{t} \leq x\right\}=0 .
$$

Case 2. Assume that $a / T \leq x<b / T$. Firstly, we will prove that

$$
\left\{\sum_{i=1}^{N_{t}} \frac{f\left(\xi_{i} \wedge T\right)}{t} \leq \frac{b}{T}\right\} \subset \bigcup_{i=2}^{\infty}\left\{\xi_{i}>T-\varepsilon\right\},
$$

for any $\varepsilon>0$ provided that $t$ is large enough. For any

$$
\gamma \in \bigcap_{i=2}^{\infty}\left\{\xi_{i} \leq T-\varepsilon\right\},
$$

when $t \geq T(2 T-\varepsilon) / \varepsilon$, it can be obtained that

$$
\begin{aligned}
\sum_{i=1}^{N_{t}(\gamma)} \frac{f\left(\xi_{i}(\gamma) \wedge T\right)}{t} & >\frac{b N_{t-T}(\gamma)}{t} \\
& \geq \frac{b((t-T) /(T-\varepsilon)-1)}{t} \\
& >\frac{b}{T} .
\end{aligned}
$$

That means

$$
\bigcap_{i=2}^{\infty}\left\{\xi_{i} \leq T-\varepsilon\right\} \subset\left\{\sum_{i=1}^{N_{t}} \frac{f\left(\xi_{i} \wedge T\right)}{t}>\frac{b}{T}\right\} .
$$

It is equivalent of

$$
\left\{\sum_{i=1}^{N_{t}} \frac{f\left(\xi_{i} \wedge T\right)}{t} \leq \frac{b}{T}\right\} \subset \bigcup_{i=2}^{\infty}\left\{\xi_{i}>T-\varepsilon\right\},
$$

provided that $t$ is larger than $T(2 T-\varepsilon) / \varepsilon$.

According to the monotonicity of uncertain distribution, for any $x \in[a / T, b / T)$, we obtain that

$$
\begin{aligned}
\lim _{t \rightarrow \infty} \Psi_{t}(x) & \leq \lim _{t \rightarrow \infty} \Psi_{t}\left(\frac{b}{T}\right)=\mathscr{M}\left\{\sum_{i=1}^{N_{t}} \frac{f\left(\xi_{i} \wedge T\right)}{t} \leq \frac{b}{T}\right\} \\
& \leq \mathscr{M}\left\{\bigcup_{i=2}^{\infty}\left\{\xi_{i}>T-\varepsilon\right\}\right\} \\
& =1-\Phi(T-\varepsilon) .
\end{aligned}
$$

Letting $\varepsilon \rightarrow 0$, we have

$$
\lim _{t \rightarrow \infty} \Psi_{t}(x) \leq 1-\Phi(T)
$$

Case 3. Assume that $x \geq b / T$. For any $\varepsilon>0$ and fixing

$$
\gamma \in \bigcap_{i=2}^{\infty}\left\{\xi_{i} \leq \frac{b}{x}-\varepsilon\right\}
$$

when

$$
t \geq \frac{b(b+x T-x \varepsilon)}{x^{2} \varepsilon},
$$

we have

$$
\begin{aligned}
\sum_{i=1}^{N_{t}(\gamma)} \frac{f\left(\xi_{i}(\gamma) \wedge T\right)}{t} & >\frac{b N_{t-T}(\gamma)}{t} \\
& \geq \frac{b}{t}\left(\frac{t-T}{b / x-\varepsilon}-1\right) \\
& >x
\end{aligned}
$$

Thus

$$
\bigcap_{i=2}^{\infty}\left\{\xi_{i} \leq \frac{b}{x}-\varepsilon\right\} \subset\left\{\sum_{i=1}^{N_{t}} \frac{f\left(\xi_{i} \wedge T\right)}{t}>x\right\} .
$$

That is

$$
\left\{\sum_{i=1}^{N_{t}} \frac{f\left(\xi_{i} \wedge T\right)}{t} \leq x\right\} \subset \bigcup_{i=2}^{\infty}\left\{\xi_{i}>\frac{b}{x}-\varepsilon\right\},
$$


provided that $t$ is large enough. Therefore, it can be obtained that

$$
\begin{aligned}
\lim _{t \rightarrow \infty} \Psi_{t}(x) & =\lim _{t \rightarrow \infty} \mathscr{M}\left\{\sum_{i=1}^{N_{t}} \frac{f\left(\xi_{i} \wedge T\right)}{t} \leq x\right\} \\
& \leq \mathscr{M}\left\{\bigcup_{i=2}^{\infty}\left\{\xi_{i}>\frac{b}{x}-\varepsilon\right\}\right\}=1-\Phi\left(\frac{b}{x}-\varepsilon\right) .
\end{aligned}
$$

Letting $\varepsilon \rightarrow 0$, for any $x \geq b / T$,we have

$$
\lim _{t \rightarrow \infty} \Psi_{t}(x) \leq 1-\Phi\left(\frac{b}{x}\right)
$$

The theorem is proved.

Theorem 10. Let $\xi_{1}, \xi_{2}, \ldots$ be a sequence of positive uncertain variables. If $\xi_{1}$ has an uncertainty distribution $\Phi_{1}$ and $\xi_{2}, \xi_{3}, \ldots$ has a common uncertainty distribution $\Phi, N_{t}$ is an uncertain renewal process with uncertain interarrivals $\xi_{1} \wedge T, \xi_{2} \wedge T, \ldots$.. Given that

$$
f(x)= \begin{cases}b, & \text { if } x<T \\ a, & \text { if } x=T\end{cases}
$$

with $0<a<b$, then

$$
\lim _{t \rightarrow \infty} E\left[\sum_{i=1}^{N_{t}} \frac{f\left(\xi_{i} \wedge T\right)}{t}\right]=\frac{a}{T}+\frac{b-a}{T} \Phi(T)+\int_{0}^{T} \frac{\Phi(x)}{x^{2}} d x
$$

Proof. Let

$$
\begin{aligned}
& \Upsilon_{1}(x)= \begin{cases}0 & \text { if } x \leq \frac{a}{T} \\
1-\Phi(T) & \text { if } \frac{a}{T}<x \leq \frac{b}{T} \\
1-\Phi\left(\frac{b}{x}\right) & \text { if } x>\frac{b}{T},\end{cases} \\
& \Upsilon_{2}(x)= \begin{cases}0 & \text { if } x<\frac{a}{T} \\
1-\Phi(T) & \text { if } \frac{a}{T} \leq x<\frac{b}{T} \\
1-\Phi\left(\frac{b}{x}\right) & \text { if } x \geq \frac{b}{T} .\end{cases}
\end{aligned}
$$

Thus

$$
\begin{aligned}
\int_{0}^{+\infty}\left(1-\Upsilon_{1}(x)\right) d x & =\int_{0}^{+\infty}\left(1-\Upsilon_{2}(x)\right) d x \\
& =\frac{a}{T}+\frac{b-a}{T} \Phi(T)+\int_{b / T}^{+\infty} \Phi\left(\frac{b}{x}\right) d x \\
& =\frac{a}{T}+\frac{b-a}{T} \Phi(T)+b \int_{0}^{T} \frac{\Phi(x)}{x^{2}} d x
\end{aligned}
$$

Note that $\lim _{t \rightarrow \infty} \Psi_{t}(x) \geq \Upsilon_{1}(x)$ by Theorem 8 and $\lim _{t \rightarrow \infty} \Psi_{t}(x) \leq \Upsilon_{2}(x)$ by Theorem 9 . According to the Fatou lemma, we can obtain

$$
\begin{aligned}
\int_{0}^{+\infty} 1-\Upsilon_{2}(x) d x & \leq \lim _{t \rightarrow \infty} E\left[\sum_{i=1}^{N_{t}} \frac{f\left(\xi_{i} \wedge T\right)}{t}\right] \\
& =\lim _{t \rightarrow \infty} \int_{0}^{+\infty} 1-\Psi_{t}(x) d x \\
& \leq \int_{0}^{+\infty} 1-\Upsilon_{1}(x) d x
\end{aligned}
$$

So we have

$$
\lim _{t \rightarrow \infty} E\left[\sum_{i=1}^{N_{t}} \frac{f\left(\xi_{i} \wedge T\right)}{t}\right]=\frac{a}{T}+\frac{b-a}{T} \Phi(T)+\int_{0}^{T} \frac{\Phi(x)}{x^{2}} d x .
$$

The theorem is proved. It follows from Theorem 10 that the optimal replacement time $T^{*}$ is just the replacement time $T$ which satisfies

$$
\min _{T}\left\{\frac{a}{T}+\frac{b-a}{T} \Phi(T)+\int_{0}^{T} \frac{\Phi(x)}{x^{2}} d x\right\} .
$$

\section{Number Example}

Let $\xi_{1}, \xi_{2}, \ldots$ be a sequence of positive uncertain variables. If $\xi_{1}$ has a lognormal uncertainty distribution $\mathscr{L} \mathscr{O} \mathscr{G} \mathcal{N}\left(e_{1}, \sigma_{1}\right)$ where $e_{1}$ and $\sigma_{1}$ are real numbers with $\sigma>0$ and $\xi_{2}, \xi_{3}, \ldots$ has a common lognormal uncertainty distribution $\mathscr{L} \mathscr{O} \mathscr{G} \mathcal{N}(e, \sigma)$ where $e$ and $\sigma$ are real numbers with $\sigma>0$, let $N_{t}$ be an uncertain renewal process with uncertain interarrivals $\xi_{1} \wedge$ $T, \xi_{2} \wedge T, \ldots$ for any $0<T<+\infty$. Given that

$$
f(x)= \begin{cases}b, & \text { if } x<T \\ a, & \text { if } x=T\end{cases}
$$

with $0<a<b$, then it follows from Theorem 10 that the expected cost over infinite time span is

$$
\begin{aligned}
\frac{a}{T}+ & \frac{b-a}{T} \Phi(T)+\int_{0}^{T} \frac{\Phi(x)}{x^{2}} d x \\
= & \frac{a}{T}+\frac{b-a}{T}\left(1+\exp \left(\frac{\pi(e-\ln T)}{\sqrt{3} \sigma}\right)\right)^{-1} \\
& +\int_{0}^{T} \frac{1}{x^{2}}\left(1+\exp \left(\frac{\pi(e-\ln x)}{\sqrt{3} \sigma}\right)\right)^{-1} d x .
\end{aligned}
$$

The optimal replacement time $T^{*}$ is just the replacement time $T$ which satisfies

$$
\begin{aligned}
\min _{T}\left\{\frac{a}{T}\right. & +\frac{b-a}{T}\left(1+\exp \left(\frac{\pi(e-\ln T)}{\sqrt{3} \sigma}\right)\right)^{-1} \\
& \left.+\int_{0}^{T} \frac{1}{x^{2}}\left(1+\exp \left(\frac{\pi(e-\ln x)}{\sqrt{3} \sigma}\right)\right)^{-1} d x\right\} .
\end{aligned}
$$




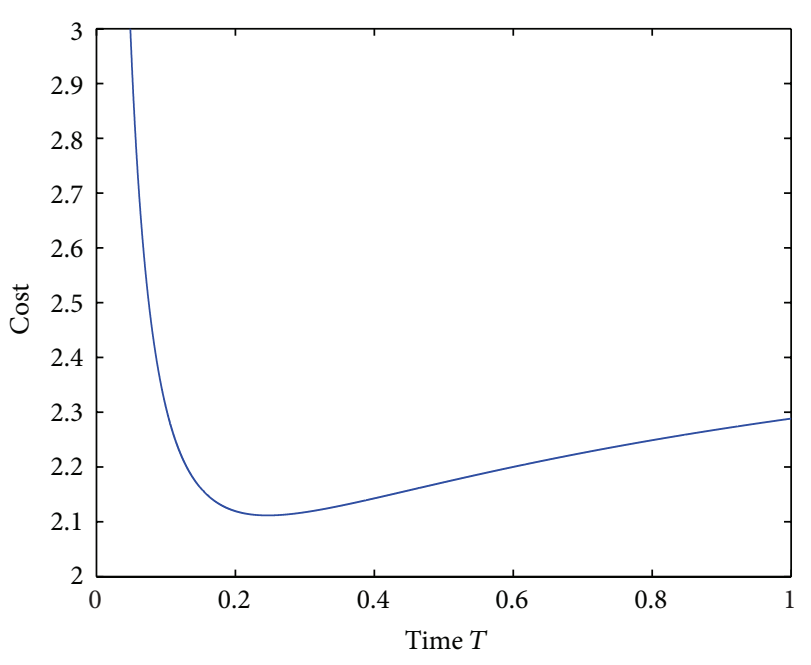

Figure 1: The expected cost function curve under the changes of time $T$.

In particular, let $e=1, \sigma=2, b=0.2$, and $a=0.1$; we give the changing trend of the expected cost function with time $T$ in Figure 1. It can be seen obviously from Figure 1 that the expected cost is monotone increasing firstly and then monotone decreasing in time $T$. We can obtain the optimal replacement time $T^{*}=0.2467$ and the minimum cost is 2.1117.

\section{Conclusions}

This paper first studied the delayed age replacement policy in uncertain environment. It gave the expected costs in infinite time span and found the optimal replacement time which minimizes the expected cost. The optimal time to replace the unit was irrelevant to the uncertain distribution of the first unit. In addition, a number example was gave.

\section{Conflict of Interests}

The authors declare that there is no conflict of interests regarding the publication of this paper.

\section{Acknowledgments}

This work was supported by the Young Scientists Fund of the National Natural Science Foundation of China (61403395), the Natural Science Foundation of Tianjin Grant 13JCYBJC39000, and Special Fund of the Civil Aviation University of China of the Fundamental Research Funds for the Central Universities under Grant no. 3122013D004.

\section{References}

[1] R. E. Barlow and F. Proschan, Mathematical Theory of Reliability, John Wiley \& Sons, New York, NY, USA, 1965.

[2] B. Fox, "Age replacement with discounting," Operations Research, vol. 14, no. 3, pp. 533-537, 1966.
[3] R. L. Scheaffer, "Optimum age replacement policies with an inceasing cost factor," Technometrics, vol. 13, no. 1, pp. 139-144, 1971.

[4] R. Cleroux, S. Dubuc, and C. Tilquin, "The age replacement problem with minimal repair and random repair costs," Operations Research, vol. 27, no. 6, pp. 1158-1167, 1979.

[5] R. Cleroux and M. Hanscom, "Age replacement with adjustment and depreciation costs and interest charges," Technometrics, vol. 16, pp. 235-239, 1974.

[6] P. J. Boland and F. Proschan, "Periodic replacement with increasing minimal repair costs at failure," Operations Research, vol. 30, no. 6, pp. 1183-1189, 1982.

[7] J. P. Jhang and S. H. Sheu, "Opportunity-based age replacement policy with minimal repair," Reliability Engineering and System Safety, vol. 64, no. 3, pp. 339-344, 1999.

[8] T. Nakagawa, Maintenance Theory of Reliability, Springer, London, UK, 2005.

[9] D. Kahneman and A. Tversky, "Prospect theory: an analysis of decisions under risk," Econometrica, vol. 47, no. 2, pp. 263-291, 1979.

[10] B. Liu, Uncertainty Theory, Springer, Berlin, Germany, 2007.

[11] B. Liu, Uncertainty Theory, Springer, Berlin, Germany, 3rd edition, 2010.

[12] K. Yao and D. A. Ralescu, "Age replacement policy in uncertain environment," Iranian Journal of Fuzzy Systems, vol. 10, no. 2, pp. 29-39, 2013, http://orsc.edu.cn/online/110906.pdf.

[13] B. Liu, "Fuzzy process, hybrid process and uncertain process," Journal of Uncertain Systems, vol. 2, no. 1, pp. 3-16, 2008. 


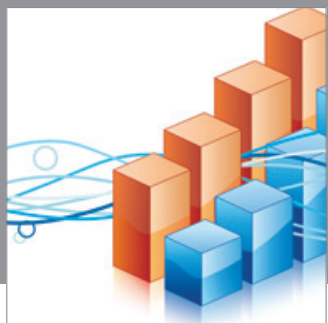

Advances in

Operations Research

mansans

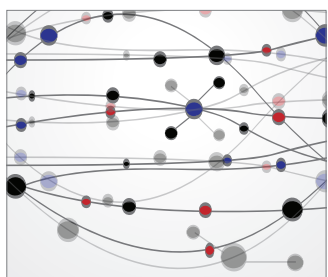

The Scientific World Journal
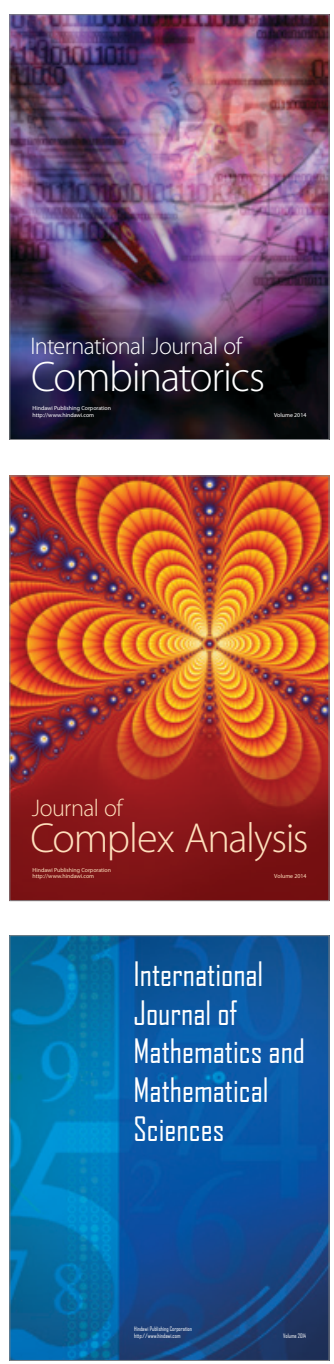
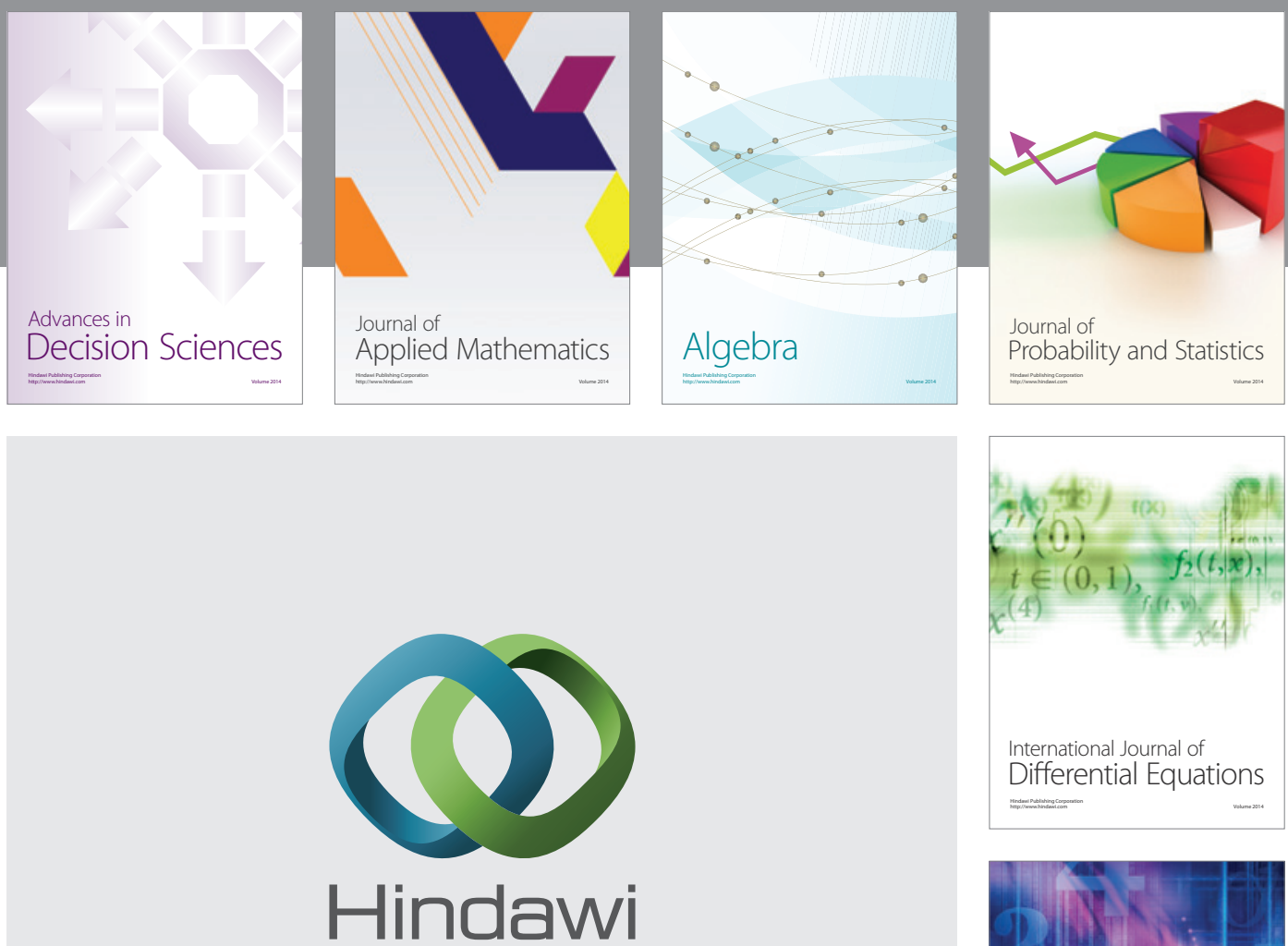

Submit your manuscripts at http://www.hindawi.com
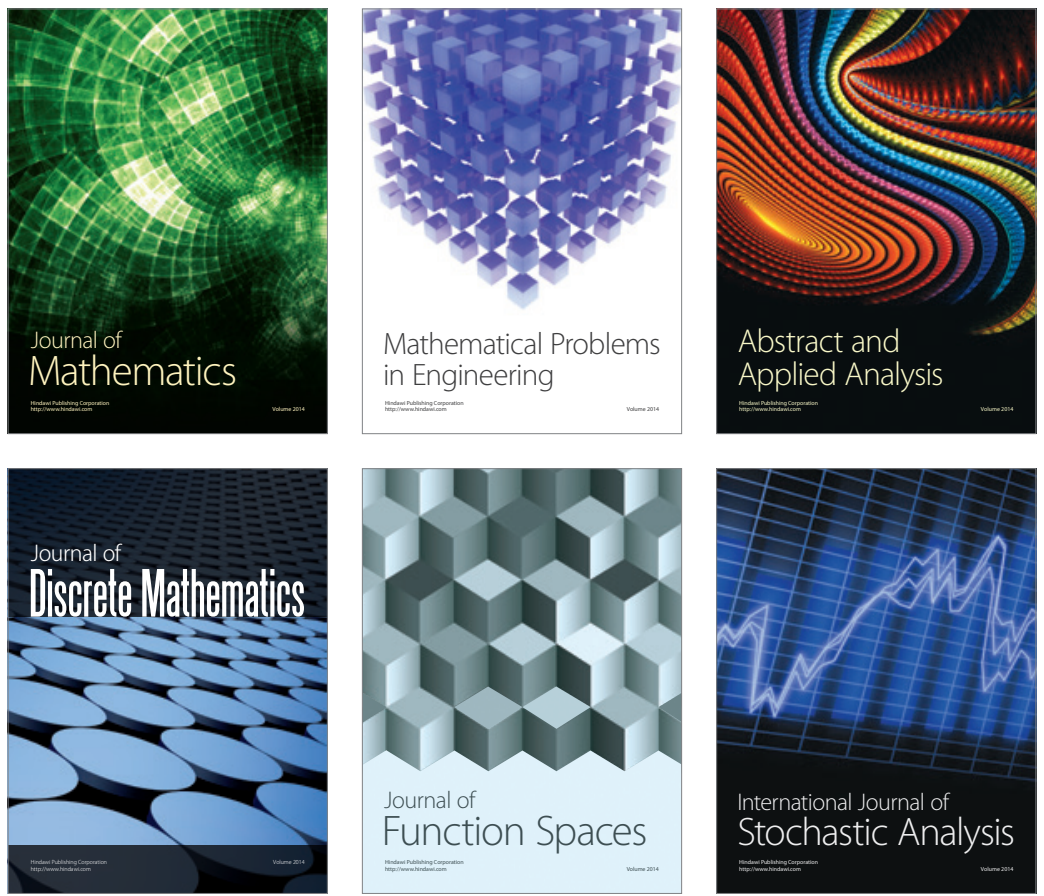

Journal of

Function Spaces

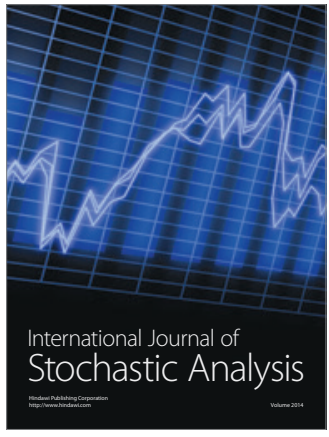

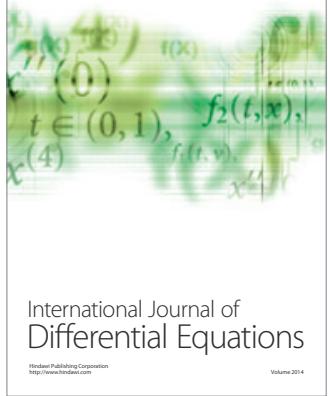
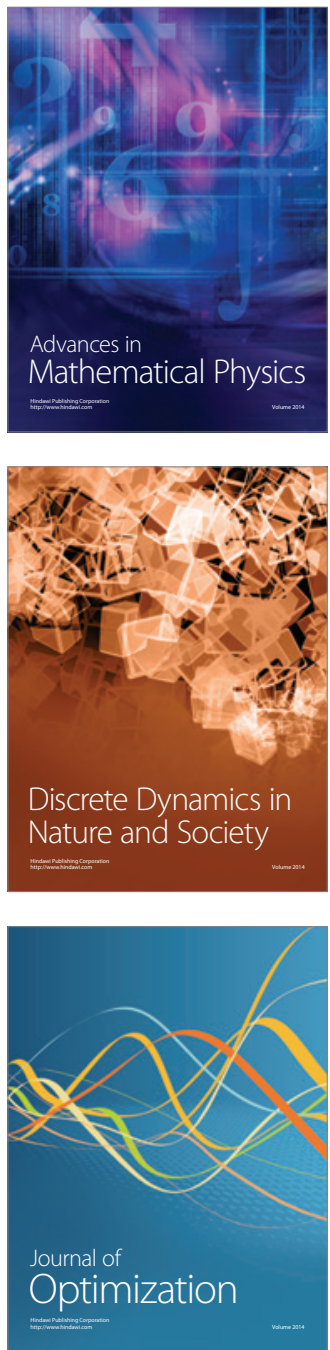